\title{
Multivariate morphometrics of the Indian honeybee in the northwest Himalayan region
}

\author{
LR Verma ${ }^{1}$, VK Mattu ${ }^{2}$, HV Daly ${ }^{3 *}$ \\ 1 International Centre for Integrated Mountain Development (ICIMOD), \\ PO Box 3226, Kathmandu, Nepal; \\ 2 Department of Bio-Sciences, HP University, Shimla, India; \\ 3 Department of Environmental Science, Policy, and Management, 201 Wellman Hall, \\ ESPM, University of California, Berkeley, CA 94720, USA
}

\begin{abstract}
Summary - Multivariate statistical analyses of 55 morphometric characters were made for collections of Apis cerana from 20 localities in the Himachal Pradesh and Jammu and Kashmir states of the northwest Himalayas. Two distinct biometric groups were observed after discriminant function analysis and cluster analysis. The groups were associated with differences in the climates of the Himachal and Kashmir regions. Previous observations were confirmed that some characters were associated with altitude and rainfall such that bees were larger and darker at higher altitudes and lower rainfall. The phenetic clustering of samples within each region corresponded in general to physiography. Principal component analysis indicated minimal contributions of general size components to overall covariation in each of the regions.
\end{abstract}

Apis cerana / Himalayas / Himachal Pradesh / Kashmir / morphometrics

\section{INTRODUCTION}

The Eastern honeybee, Apis cerana Fabricius, occupies a distribution extending from Afghanistan to Japan and thence southeast in Asia to the Wallace Line. This study is focused on collections made at 20 localities in the Himachal region (state of Himachal Pradesh) and Kashmir region (state of Jammu and Kashmir) of the northwest Himalayan Region (see map in Mattu and Verma, 1983). Univariate analyses of bees from these collections have been published and provide additional information (Mattu and Verma, 1983, 1984a,b; Verma and Mattu, 1982; Verma et al, 1984). In this study, certain univariate analyses are re-examined by analysis of variance (ANOVA) and summarized, and multivariate discriminant function analysis (DFA) and principal component analysis (PCA) are performed.

\footnotetext{
${ }^{*}$ Correspondence and reprints
} 


\section{MATERIALS AND METHODS}

Collections of worker bees were made from 4-5 wild colonies at each of 20 localities. The total number of bees from each locality and other information are given in table I (see also Mattu and Verma, 1983). The 55 characters were those for which univariate analyses were previously reported by Mattu and Verma (1983, 1984a, b). Code numbers for the characters and brief descriptions of the measurements are provided in table II.

Computations were performed on an IBM 3090 computer at the University of California, Berkeley. For the discriminant analyses the programs DISCRIMINANT (prior probabilities equal; minimum tolerance in stepwise procedure was 0.001 ) and FACTOR from the Statistical Package for the Social Sciences (Norusis, 1985) was used. The program NTSYS-PC (Rohlf, 1987) was used to perform unweighted pair-group cluster analyses with arithmetic averages (UPGMA) as described by Sneath and Sokal (1973). All other computations were made with special programs written by HVD and performed on an IBM-AT personal computer.

The statistical analyses were performed in four phases in a manner similar to that described by Singh et al (1990). The purpose of the first phase was to make an initial DFA of the 20 localities to determine how the collections of bees from the 20 localities could be clustered into a smaller number of groups. The measurements of all characters for the collections were grouped by locality and entered as 20 groups in a DFA. The program included a univariate ANOVA for each character over the 20 groups. The extent to which the DFA discriminated among the localities was evaluated by reclassifying the 1184 individual bees with the coefficients from the analysis. Each bee was assigned to the group for which the bee had the highest probability of membership. With 20 groups of equal prior probability, $5 \%$ could be correctly classified by chance alone.

The mean value of each character at each locality was then used to compute the coordinates of the centroids for each locality with respect to the 19 canonical discriminant functions. From the coordinates, the square roots of the Euclidean distances between each pair of localities were computed. The smaller the distance, the more similar are the biometries of the bees collected at two localities. A hierarchic UPGMA cluster analysis was made of the Euclidean distances among the 20 localities (Sneath and Sokal, 1973). On this basis, two major biometric groups were recognized.

The purpose of the second phase of analyses was to evaluate the discrimination between the two major biometric groups using all $\mathbf{5 5}$ characters. The measurements for the collections were regrouped into the two biometric groups and entered in a second series of DFAs. ANOVA for each character was performed again, now for the two groups. Two DFA methods were used: the 'direct' method in which all characters were included and the 'stepwise with Wilks criterion' method in which certain characters were automatically eliminated if they failed to meet certain critical values. The discrimination between the groups was evaluated by reclassifying each bee into one of the two biometric groups. With two groups of equal prior probability, $50 \%$ of the bees could be correctly classified by chance alone. Finally, the odd numbered samples were held as a test set and only the even numbered samples were used as data for the DFA. Then all samples were classified into the two groups. Next, the even numbered samples were held as a test set and so forth. The classification of the test sets in each case provided further evidence of the effectiveness of discrimination.

The purpose of the third phase of the analyses was to evaluate the contributions of the different characters to the discrimination of the two biometric groups. This was done by two methods: 1) by ranking of the absolute values of the standardized discriminant function coefficients; and 2) ranking the absolute values of the correlations of the 55 characters with the single discriminant function. As a result of these comparisons, a reduced number of characters was selected to provide a quick method for identification of the biometric groups. The final choice was supported by the procedure described above where odd or even samples were held as test sets. ANOVAs were also made for the 55 characters within each biometric group to examine the differences in the characters within the two groups.

The purposes of the fourth phase were: 1) to determine the relationships of the morphometric characters to altitude and rainfall; and 2) to determine the contribution of a general size component to overall variation within each biometric group. First, univariate product-moment correlations $(r)$ were computed for altitude and rainfall at the localities (table I) versus the values of the 55 morphometric characters for individual bees. 
Second, a PCA was performed with altitude and rainfall included as variables with the 55 characters for individual bees. Because the two biometric groups were so different, the computations of correlations and PCA were carried out within each biometric group rather than overall.

\section{RESULTS}

\section{Discriminant analysis}

In the first phase, the initial DFA indicated that a majority of the bees from each of the 20 localities were closer to other bees from the same locality than to bees from other localities when placed in a 19-dimensional morphometric space. This was shown by the reclassification of the individual bees into the 20 groups by two DFAs with different procedures. The direct method, where all 55 characters were included, gave an overall average of $61.15 \%$ of bees correctly classified and the stepwise method gave $61.23 \%$ correctly classified. The univariate ANOVA indicated that 48 of the 55 characters over all 20 localities were highly significant (significance probability $<0.001$; see table VI for list).

The biometric relationships of the bees from the 20 localities are shown in the phenogram (fig 1). Two distinct groups are evident: samples 51 to 62 from Himachal region form one biometric group, and samples 63 to 70 from Kashmir region form a second biometric group. The two biometric groups are so distinct that clearer pictures of the three-dimensional relationships of the samples in each region are best shown in separate illustrations (figs 2,3).

The second phase was a DFA of the two biometric groups with all 55 characters. When all samples were entered as data, the overall classification rate was $99.9 \%$ regardless of whether all 55 characters were used in the direct method or just the 39 characters selected by the stepwise procedure were used. Because the two methods gave essentially the same result, we provide here the statistics from the direct method: unstandardized coefficients for use with raw measurements and standardized coefficients for use with standardized measurements (table IV; see table III for total means and standard deviations). The ANOVA of the two groups gave highly significant $F$ values for 45 of the 55 characters (significance probability $<0.001$; see table VI for list).

In the third phase, the characters with standardized discriminant coefficients of absolute value $>0.35$ (an arbitrarily chosen value) on the single function were characters 48,25 and 46. These characters also had correlations with the function of absolute value $>0.33$ (see table IV). When only characters 48 and 25 were used in a DFA between the biometric groups and all bees were included as data, the overall percentage of correct classification was $99.9 \%$. When samples were split into test and data sets, the percentages of correct classifications for the data sets were $100 \%$ (even numbered localities) and $99.8 \%$ (odd numbered localities). For the corresponding test sets, $99.8 \%$ and $100 \%$, respectively. Table $\checkmark$ provides statistics for a quick classification of new samples with only two characters. When classification of new samples into the two biometric groups is critical, then the use of all 55 measurements is recommended.

\section{Variation and correlation of characters}

The several ANOVAs that were performed for different groupings of the data indicate that forewing characters 10 and 14 , tongue characters 34-36, and antenna character 53 were not different among the samples or groups, while forewing characters 2,4 , 7,8 , and 17 , hindwing character 29 , and abdomen character 41 consistently differed between the regions and among the samples within each region (table VI). ANOVAs 
within each region indicated that 31 characters of bees of the Himachal region and 16 characters of bees from the Kashmir region were different among the samples. Bees within the latter region, therefore, exhibited the least differences among the samples.

The correlations of rainfall and altitude with morphometrics of individual bees were examined separately within each region by univariate and multivariate analysis (tables VI, VII). A univariate correlation existed between rainfall versus altitude among localities of the Himachal region ( $r=-0.38$, significance probability $=0.00$ ), but no correlation existed among the localities of Kashmir region ( $r=0.02$, significance probability $=0.33$ ). These relationships also appeared in the PCAs where altitude and rainfall jointly contributed strongly to $P C 1$ in the analysis of the Himachal region, but were unrelated and divided in their contributions to PC1-PC3 in the analysis of the Kashmir region (table VII).

For bees of the Himachal region, 35 morphometric characters had significant correlations with altitude (two-tailed significance probability $<0.01$ ). All but two of these were positive. The highest correlations ( $>0.3$, an arbitrary value) were found for forewing characters 2 and 5; hindwing character 24; hind leg character 30; and abdomen characters 41,50 , and 51 . Fifteen characters had significant, negative correlations with rainfall of which abdomen characters 41 and 50 were $<-0.17$.

For bees of the Kashmir region, 28 characters were correlated with altitude; all but three were positive. Among the highest were forewing character 7 and hindwing character 26 at $>0.29$. Seven characters were correlated with rainfall, of which five were negative. Forewing character 6 and abdomen character 47 were highest at $<-0.18$.

In summary, most correlations with altitude and rainfall within a region were found in wing, leg, and abdomen characters, while relatively few were found among tongue and antenna characters. Most correlations with altitude were positive and most with rainfall were negative. In other words, higher altitude and lower rainfall were correlated with larger size within each region. Twenty-two characters were consistently correlated with altitude in both groups, but only character 4 was correlated with rainfall in both groups.

As observed previously, bees of the Kashmir region are larger in length and width measurements than those of the Himachal region (Mattu and Verma, 1983, 1984a, b). This was confirmed by comparing the means of the characters (table III) after excluding characters 9-19 which are angles and characters $10,14,18,21,26,34,35$, 36,43 , and 53 which are not significantly different between the groups. The exceptional characters were 38,40 , and 48 , where on the average, bees of the Himachal region are larger.

The PCAs of morphometrics within each region (table VII) shared certain features in common: about $4 \%$ of the variation was accounted for by PC1 in each analysis and $<2 \%$ was accounted for by each of the remaining components. As a result, the cumulative percentages of variances explained by components 1-23 were only $55.9-58.9 \%$. Altitude had the highest correlations of all variables with PC1. Morphometric variables that had higher correlations with $\mathrm{PC} 1$ in both analyses were forewing characters 1, 5 and 29; hind leg characters 30 and 31; and abdomen character 41 . Otherwise, the two PCAs shared little in common with regard to the distribution of the higher correlations among the components.

\section{DISCUSSION}

Two major biometric clusters emerged when all 20 samples were analyzed in a single DFA (fig 1). The 8 samples from the Kashmir region formed one biometric group and 
the 12 samples from 1 the Himachal region formed the second group. The morphometric 'gap' between the groups was quite distinct. Samples from localities along the boundary between the regions did not appear to form a transitional cline. For example, bees from sample 63 (table I) taken at a lower elevation in western Kashmir region did not cluster with bees taken at lower elevations in the western Himachal region. Vice versa, bees from sample 58 taken at a higher elevation in the northern Himachal region did not cluster with bees from sample 64 , also taken at a high elevation, in the southern Kashmir region. The overall lack of similarity in the distribution of correlations with the first three principal components of morphometric covariation in each region is further evidence of the difference between bees in the two regions.

Although no striking physiographic feature separates these regions, a major climatic boundary does coincide with the political boundary between the states. The rainfall statistics alone do not reveal this climate difference (table I). However, based on precipitation effectiveness (total monthly precipitation divided by total monthly evaporation), the climate classification of Takahashi and Arakawa (1981) shows the collection sites of the Kashmir region are in a zone of dry sub-humid climate while those of the Himachal region are in zones of moist sub-humid or humid climates. Analysis of future collections along this climatic boundary will be necessary to discover where the transition from one biometric type to the other occurs or if the two groups coexist sympatrically. In the latter case, an appropriate taxonomic action would be in order.

The two biometric groups were distinguished by significant differences in 45 of the 55 characters. Bees of the Kashmir region were larger on the average than those of the Himachal region in most length and breadth measurements; exceptions were characters 38 (length of postmentum) and 48 (length of wax mirror on 3rd sternite) in which bees of the Kashmir region are smaller. Bees of the Kashmir region also had wider bands of dark color on the third and fourth terga and a narrower light band on the third tergite. However, the characters of the tongue were complex: length and breadth of prementum and total tongue length were not different between the groups, length of labial palp and glossa were larger in the Kashmir region, while length of the postmentum was smaller in the Kashmir region.

Within each region, correlations also existed between morphometric characters and altitude and rainfall. Larger size in a majority of length and breadth measurements was associated with higher altitude and lower rainfall. These results agree in general with previous analyses, but differ in some details because of differing computational procedures (Mattu and Verma, 1983, 1984a, b; Verma and Mattu, 1982; Verma et al, 1984). Except for the correlations with altitude, and to a lesser extent with rainfall, covariation among the morphometric characters themselves as revealed by PCA was remarkably low. This resulted in a minimal contribution of 'general size' to the overall covariation among the samples within each region. The first component usually accounts for a much larger percentage of variance in morphometric studies (Daly, 1992).

Within each biometric group, clusters of samples in morphometric space were often, but not always, close to each other geographically. In the Kashmir region, a close correspondence existed between the minor clusters (figs 1,3) and physiographic features: sample 68 from the Kishangana Valley, sample 69 from the Sindh Valley, and sample 70 from the Drass Valley, are all from contiguous river valleys at altitudes above $2300 \mathrm{~m}$. Another cluster of samples 65,66 , and 67 was taken in the Kashmir Valley, just to the south of the first cluster and at altitudes of $1700-2000 \mathrm{~m}$. All six 
samples were united in a larger cluster at phenon 5 and all were taken north of the Pan Panjal Range which produces a modified monsoon climate. Samples 63 and 64 were isolated morphometrically and the collection sites were separated geographically. However, these two sites share lower altitudes and the normal monsoon climate which prevails south of the Pan Panjal Range and in the Chenab River Valley that penetrates the range.

In the Himachal region, most samples in the minor clusters can be related to physiography and the geographic proximity of morphometrically related samples, but some samples cannot. The cluster of samples 59 , 60,61 , and 62 were taken from a zone of humid climate, dominated by the Sutlej River valley, and stretching from Simla to the Kinnaur region. The remaining samples of 1 the Himachal region were from the moist sub-humid zone or along the its boundary with the humid zone. The cluster of sam- ples $52,53,55$, and 56 was taken in the region of the Beas River and limited on the north by the Dhaula Dhar Range. An exception was sample 54 which was morphometrically close to sample 56 , but was taken much farther south. Likewise, samples 57 and 58 were clustered morphometrically, but were not close geographically. Finally, sample 51, isolated from other samples morphometrically, was taken at the lowest altitude.

\section{ACKNOWLEDGMENTS}

The authors are extremely pleased to contribute to this special issue in honor of Professor Ruttner. This research was supported in part under Grant No: 367-5600-G-SS-1147-00, Programme in Science and Technology Cooperation, Office of Science Adviser, US Agency for International Development. Funds for computation were provided by the Agricultural Experiment Station of the University of California.

\title{
Morphometrische Untersuchung an Indischen Honigbienen aus dem Gebiet des nordwestlichen Himalayas
}

\begin{abstract}
Zusammenfassung - Es wurden Bienenproben der Art Apis cerana an 20 verschiedenen Orten in den Staaten (Provinzen) des Nordwest Himalaya gesammelt: Himachal Pradesh, Jammu und Kashmir. Von jeder Biene wurden 55 Merkmale gemessen und mit multivariaten statistischen Methoden bearbeitet. Mit Hilfe der Diskriminanz- und Clusteranalyse konnten zwei verschiedene morphometrische Gruppen unterschieden werden, die jeweils mit klimatischen Unterschieden assoziiert waren. Bereits bekannte Befunde konnten bestätigt werden. Bei großer Höhe und geringerem Niederschlag waren die Bienen größer und dunkler. Die Verteilung der Proben in phenetische Cluster innerhalb jeder Region zeigte eine allgemeine Übereinstimmung mit den topographischen und klimatischen Bedingungen. Allgemeine Größenmerkmale trugen, wie die Principal Component Analysis zeigte, nur sehr wenig zur Gesamtkovarianz in jeder Region bei.
\end{abstract}




\section{EINLEITUNG}

Die 'östliche' Honigbiene Apis cerana Fabricius hat ein großes Verbreitungsgebiet, das sich von Afghanistan bis Japan und von dort südöstlich bis zur Wallace Linie erstreckt. Diese Untersuchung beschränkt sich auf 20 Orte in den beiden Regionen Himachal (Himachal Pradesh) und Kashmir (Jammu, Kashmir), die im nordwestlichen Himalaya liegen (Karte bei Mattu und Verma, 1983). Einfache Varianzanalysen von dieser Bienensammlung sind bereits publiziert (Mattu und Verma, 1983, 1984a,b; Verma und Mattu, 1982; Verma et al, 1984). Hier werden diese Ergebnisse mit Hilfe von einer Varianzanalyse (ANOVA) überprüft und zusammengefaßt. Eine weitergehende Bearbeitung der Daten wurde mit Hilfe einer multivariaten Diskriminanzanalyse (DFA) und einer 'principal component analysis' (PCA) durchgeführt.

\section{MATERIAL UND METHODE}

Bienenarbeiterinnen wurden von 4-5 wildiebenden Bienenvölkern an jedem der 20 Orte gesammelt. Die Gesamtzahl der Bienen findet sich in Tabelle I (siehe auch Mattu und Verma, 1983). Die 55 gemessenen Merkmale stimmen mit der Arbeit von Mattu und Verma (1983, 1984a,b) überein. Eine Kurzbeschreibung der Merkmale und die Codenummer finden sich in Tabelle II.

Es wurden schrittweise verschiedene statistische Verfahren angewendet und die Bienenproben morphometrisch aufgegliedert. Angaben über die genaue Vorgehensweise und die benutzte Software finden sich in der englischen Originalfassung.

\section{ERGEBNISSE}

\section{Diskriminanzanalyse}

In der ersten Phase der DFA wurden die Bienen der 20 Orte in einem 19-dimensio- nalen morphometrischen Raum verteilt. Dabei zeigte sich, daß bei der Mehrzahl der Bienen jeweils im Vergleich mit anderen Orten eine größere Nähe zu Bienen des gleichen Ortes bestand. Das wurde mit Hilfe von zwei verschiedenen DFA-Methoden im Zuge einer Reklassifizierung der einzelnen Bienen nachgewiesen. Die direkte Methode, bei der alle 55 Merkmale berücksichtigt wurden, ergab eine durchschnittlich $61,15 \%$ richtige Zuordnung. Bei der schrittweisen DCA wurden $61,23 \%$ korrekt klassifiziert. Die ANOVA zeigte, daß 48 von 55 Merkmalen bei allen Orten hoch signifikant waren $(P<0,001$, Tabelle VI für die Liste).

Die biometrische Verwandtschaft der Bienen zwischen den 20 Orten werden in einem Phenogramm gezeigt (Abb 1). Zwei unterschiedliche Gruppen werden nachgewiesen. Die Proben 51 bis 62 aus der Himachalregion und die Proben 63 bis 70 aus Kashmir. Diese zwei biometrischen Gruppen unterscheiden sich so stark, daß die Zuordnungen innerhalb der Regionen klarer in separaten dreidimensionalen Schaubildern dokumentiert werden können (Abb 2).

In der zweiten Phase der statistischen Auswertung wurde eine DFA der zwei biometrischen Gruppen mit allen 55 Merkmalen vollzogen. Die Gesamtrate der richtigen Klassifizierung war $99,9 \%$ unabhängig davon, ob alle 55 Merkmale im direkten Verfahren eingesetzt wurden oder nur die 39 Merkmale, die durch das schrittweise Verfahren ausgewählt worden waren. Weil beide Methoden im Prinzip zu den gleichen Ergebnissen führen, geben wir nur die Statistik für das direkte Verfahren an (Tabelle IV, Tabelle III für die Mittelwerte und Standardabweichungen). Weiter ergab die ANOVA der beiden Gruppen hoch signifikante F-Werte für 45 der 55 Merkmale ( $P$ $<0,001$, Tabelle VI).

In der 3. Phase wurden die Merkmale mit einem standardisierten Diskriminationskoefizienten $>0,35$ (eine willkürlich festge- 
legte $\mathrm{Größe)} \mathrm{bestimmt.} \mathrm{Es} \mathrm{waren} \mathrm{die} \mathrm{Merk-}$ male 48, 25 und 46, die zugleich eine absolute Korrelation mit der Funktion von $>0,33$ hatten (Tabelle IV). Wenn ausschließlich die Merkmale 48 und 25 in einer DFA zwischen beiden biometrischen Gruppen und allen Bienen benutzt wurden, betrug der Prozentsatz der richtigen Klassifizierung $99,9 \%$. Bei Trennung der Proben in Testund Datensets war die richtige Klassifizierung für die Datensets $100 \%$ (für gerade Probennummern) und $99,8 \%$ (ungerade Probennummern). Für die zugehörigen Testsets errechneten sich $99,8 \%$ und $100 \%$. In Tabelle $V$ finden sich die Angaben für das statistische Schnellverfahren zur Klassifizierung von neuen Proben mit nur zwei Merkmalen. Wenn allerdings die Klassifizierung von neuen Proben in die beiden biometrischen Gruppen nicht klar ist, soll eine Berücksichtigung von allen 55 Merkmalen empfohlen werden.

\section{Variationsbreite und Korrelation}

Es wurden ANOVAs mit mehreren verschiedenen Gruppierungen von Merkmalen durchgeführt. Dabei stellte sich heraus, daß die Vorderflügelmerkmale 10 und 14 , die Rüsselmerkmale 34-36 und das Antennenmerkmal 53 sich nicht zwischen den Proben oder den Gruppen unterschieden. Dayegen zeigten sich bei folgenden Merkmalen konsistente Unterschiede zwischen den Regionen und den Proben innerhalb der Regionen: Vorderflügelmerkmal 2, 4, 7, 8 und 17, Hinterflügelmerkmal 29 und Abdomenmerkmal 41 (Tabelle VI). ANOVAs innerhalb der beiden Regionen zeigten, daß 31 Merkmale in der Himachalregion und 16 Merkmale in Kashmir zwischen den Proben verschieden waren. Damit wurde nachgewiesen, daß die Unterschiede zwischen den Kashmirproben kleiner sind als zwischen den Himachalproben.
Die Korrelationen zwischen Niederschlagsmenge und Höhenlage mit der Morphometrie einzelner Bienen wurde getrennt für jede Region geprüft (Tabellen VI und VII). Zunächst zeigte sich eine Korrelation von Niederschlag und Höhenlage für die Orte der Himachalregion $(r=-0,38 ; P=$ $0,00)$. Für Kashmir konnte diese Korrelation nicht gezeigt werden $(r=0,02 ; P=$ 0,33 ). Diese Beziehungen traten deutlich bei den PCAs in Erscheinung. Bei der Analyse der Himachalregion trugen Niederschlag und Höhe gemeinsam sehr stark zu PC1 bei. In Kashmir waren dagegen beide Faktoren unabhängig voneinander und teilten sich unter PC1-PC3 auf (Tabelle VII).

Für die Bienen der Himachalregion zeigten 35 Merkmale eine signifikante Korrelation zur Höhenlage (zweiseitige Verteilung, $P<0,01$ ), wobei mit Ausnahme von zwei Merkmalen alle Korrelationen positiv waren. Die höchsten Korrelationen ( $>0,3$; willkürlicher Wert) ergaben sich für den Vorderflügel (2 und 5), den Hinterflügel (24), das Hinterbein (30) und das Abdomen (41, 50 und 51). Bei 15 Merkmalen ergaben sich signifikante negative Korrelationen mit dem Niederschlag, wobei die Abdomenmerkmale 41 und 50 eine Korrelation $<-0,17$ zeigten.

Bei den Kashmir-Bienen waren 28 Merkmale mit der Höhe korreliert, wobei nur 3 Merkmale eine negative Korrelation hatten. Die höchsten Werte $(>0,29)$ errechneten sich für Vorderflügelmerkmal 7 und Hinterflügelmerkmal 26. Mit der Niederschlagsmenge waren 7 Merkmale korreliert, wobei in 5 Fällen negative Werte gefunden wurden. Im Vorderflügel zeigte Merkmal 6 und am Abdomen Merkmal 47 eine Korrelation $<-0,18$.

Insgesamt wurden die häufigsten Korrelationen mit Höhenlage und Niederschlagsmenge bei Flügel-, Bein- und Abdominalmerkmalen gefunden, beim Rüssel und der Antenne dagegen traten nur selten solche Korrelationen auf. Die meisten Korrelationen mit der Höhe waren positiv und 
die mit der Niederschlagsmenge negativ. Mit anderen Worten: größere Höhe und weniger Niederschlagsmenge waren mit größeren Körpermaßen der Bienen in beiden Regionen korreliert. Mit der Höhe zeigten 22 Merkmale eine konsistente Korrelation in beiden Gruppen, wohingegen nur 4 Merkmale mit der Niederschlagsmenge entsprechend negativ korrelierten.

Wie bereits früher beschrieben, haben die Bienen der Kashmirregion größere Körpermaße als die der Himachalregion (Mattu und Verma, 1983, 1984a,b). Nach Ausschluß der Merkmale 9-19 (Winkelmaße) und $10,14,18,21,26,34,35,36,43$ und 53 (nicht signifikant unterschiedlich zwischen den Gruppen) konnte der vorstehende Befund bestätigt werden. Die Merkmale 38 , 40 und 48 waren dagegen im Durchschnitt bei den Himachalbienen größer.

Die PCAs innerhalb der Regionen (Tabelle VII) zeigten gewisse Gemeinsamkeiten: ca 4\% der Varianz ergab sich für $P C 1$ und für jede der verbliebenen Faktoren errechnete sich eine Varianz von $<2 \%$. Als Ergebnis davon ergaben die kumulativen Prozentwerte für die Faktoren (PC) 1-23 nur $55,9 \%-58,9 \%$. Die höchste Korrelation zeigte sich bei PC1 für die Höhenlage. Die morphometrischen Varianzen, die bei beiden Analysen die höchsten Korrelationen ergaben, waren die Vorderflügelmerkmale 1,5 und 29, die Hinterbeinmerkmale 30 und 31 sowie Merkmal 41 (Abdomen). Darüberhinaus gab es zwischen den beiden PCAs nur wenige Übereinstimmungen.

\section{DISKUSSION}

Die beiden biometrischen Cluster ergaben sich bei einer ersten DCA (Abb 1): Die 8 Proben der Kashmirregion bildeten die erste biometrische Gruppe und die Proben aus der Himachalregion schlossen sich zu einer zweiten biometrischen Gruppe zusammen. Der 'morphometrische' Abstand zwischen beiden Gruppen ist sehr deutlich ausgeprägt. Die Proben entlang der Grenze zwischen beiden Regionen zeigten keine Übergänge. Probe 63, die aus einer geringen Höhe im westlichen Kashmir stammt, wurde nicht den Bienen aus niedrigen Höhenlagen im Himachal zugeordnet. Desgleichen bildeten auch die Bienen von Probe 58 aus großer Höhenlage der Himachalregion kein gemeinsames Cluster mit Bienen (Probe 64) aus dem südlichen hochgelegenen Teil von Kashmir. Die Unterschiede zwischen den Bienenproben beider Regionen werden zusätzlich durch den Mangel von generellen Ähnlichkeiten bei der Verteilung von Korrelationen zwischen den ersten 3 PCs der morphometrischen Kovarianz unterstrichen.

Beide Regionen unterscheiden sich wenig in der topographischen Ausgestaltung, jedoch gibt es eine klimatische Grenze zwischen der Himachal- und der Kashmirregion. Die Niederschlagsmenge allein zeigt nicht die vollen Unterschiede (Tabelle I). Erst die effektive Niederschlagsmenge (monatliche Gesamtniederschlagsmenge bezogen auf die Verdunstungsrate) zeigt die klimatische Einordnung der Sammelorte in Kashmir in eine 'trockene, wenig feuchte' Zone während die Sammelorte in der Himachalregion zu der feuchten Zone nach Takahashi und Arakawa (1981) gehören. Um zu entscheiden, ob eine Cline (= Reihe von Übergangsformen) zwischen beiden Bienentypen existiert, müßten weitere Bienenproben an der klimatischen Grenzlinie gesammelt werden. Sollten dagegen beide biometrische Gruppen nebeneinander vorkommen, so wäre eine taxonomische Auftrennung gerechtfertigt.

Beide biometrische Gruppen unterscheiden sich signifikant in 45 von 55 Merkmalen. Im Durchschnitt waren die Bienen der Kashmirregion größer als die der Himachalregion. Ausnahmen davon fanden sich bei der Länge des Postmentums (38) und der Länge des Wachsspiegels (48), die 
bei den Kashmirbienen kleiner sind. Die schwarzen Abdominalbänder (3. und 4.Tergit) waren gleichfalls breiter bei Bienen aus Kashmir. Bei den Rüsselmerkmalen waren die Zusammenhänge nicht einheitlich: Länge und Breite des Prämentums und die Gesamtlänge des Rüssels waren nicht unterschiedlich zwischen den Gruppen. Für die Länge des Labialpalpus und der Glossa ergaben sich größere Werte für die Kashmirregion, wohingegen die Länge des Postmentums dieser Bienen kleiner war. Innerhalb der Region ergaben sich jeweils Korrelationen zwischen mehreren Merkmalen und Niederschlagsmenge bzw Höhenlage. Dabei waren größere Längen und Breiten von Körpermaßen mit größerer Höhe und weniger Niederschlag verbunden. In der Tendenz stimmen die Ergebnisse mit der voherigen Analyse von Mattu und Verma (1983, 1984a,b), Verma und Mattu (1982) und Verma et al (1984) überein und es gibt wegen der hier angewandten neuen Dateriverarbeitung Unterschiede in einigen Einzelheiten, Die Kovarianz zwischen den morphometrischen Werten, ausgenommen die Korrelation mit der Höhe und in geringerem Umfang der Niederschlagsmenge, war bemerkenswert niedrig wie die PCA zeigte. Auf diese Weise trug dann auch die 'allgemeine Größe" nur in sehr geringem Umfang zur Gesamtkovarianz innerhalb der Proben einer Region bei. Bei morphometrischen Untersuchungen repräsentiert in der Regel die erste Komponente einen sehr viel höheren Prozentsatz der Gesamtvarianz (Daly, 1992).

In der biometrischen Gruppe lagen häufig geographisch benachbarte Proben auch im Cluster dicht beieinander. In der Kashmirregion gab es eine gute Übereinstimmung zwischen kleineren Clustern und Geländeformationen: Probe 68 aus dem Kishangana Tal, Probe 69 aus dem Sindh Tal und Probe 70 vom Drass Tal stammen alle aus hochgelegenen (über $2300 \mathrm{~m}$ ), zusammenhängenden Flußtälern. Ein weiteres Cluster (65,
$66,67)$ wurde im Kashmir Tal in einer Höhe von 1700 - $2000 \mathrm{~m}$ gesammelt, unmittelbar südlich vom vorherigen Cluster. Alle 6 Proben bilden zusammen ein größeres Cluster und alle diese Bienen wurden nördlich der Pan Panjal Range gefunden, wo ein abweichendes Monsunklima herrscht. Die Proben 63 und 64 liegen sowohl morphometrisch als auch geographisch isoliert. Sie stammen jedoch von Orten niedriger Höhenlage, in denen das normale Monsunklima dominiert.

In der Himachalregion können die Mehrzahl der Proben in den kleineren Clustern mit Geländeformation oder mit geographischer Nachbarschaft in Verbindung gebracht werden. Die Proben 59, 60, 61, 62 bilden ein Cluster, zugleich stammen sie aus einer feuchten Klimazone, die vom Sutley Tal beherrscht wird und die sich von Simla in die Kinnaurregion erstreckt. Die Proben 52, $53,55,56$ bilden ein weiteres Cluster und stammen aus der Gegend des Beas Flusses, die im Norden durch die Berge der Dhaula Dhar Range begrenzt wird. Als Ausnahme muß Probe 54 gelten, die morphometrisch nahe bei Probe 56 lag, aber viel weiter südlich gesammelt wurde. Auch die Proben 57 und 58 lagen morphometrisch dicht zusammen, stammten aber von entfernt gelegenen Orten. Probe 51, die morphometrisch von den anderen Proben getrennt liegt, stammt von der niedrigsten Höhenlage.

\section{DANKSAGUNG}

Die Autoren sind glücklich, daß sie mit dieser Arbeit zum Sonderheft der Apidologie zu Ehren von Professor Ruttner beitragen können.

Übersetzung: N Koeniger.

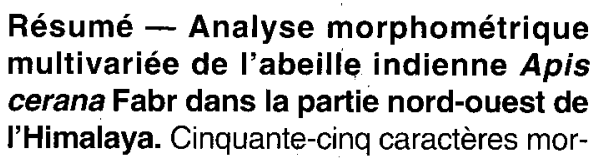


phométriques (tableau II) d'abeilles Apis cerana provenant de 20 localités des États d'Himachal Pradesh et de Jammu et Cachemire dans le nord-ouest de l'Himalaya ont été soumis à des analyses statistiques multivariées. Deux groupes ont été identifiés après analyse discriminante et analyse de groupe (fig 1). Les groupes sont liés aux différences climatiques des 2 régions. Des observations antérieures, selon lesquelles certains caractères étaient associés à l'altitude et à la pluviométrie, ont été confirmées. La plupart des corrélations avec l'altitude et la pluviométrie à l'intérieur d'une région ont été trouvées pour les caractères de l'aile, de la patte et de l'abdomen, alors que les corrélations avec les caractères de la langue et de l'antenne sont peu nombreuses (tableaux VI et VII). La plupart des corrélations avec l'altitude sont positives et celles

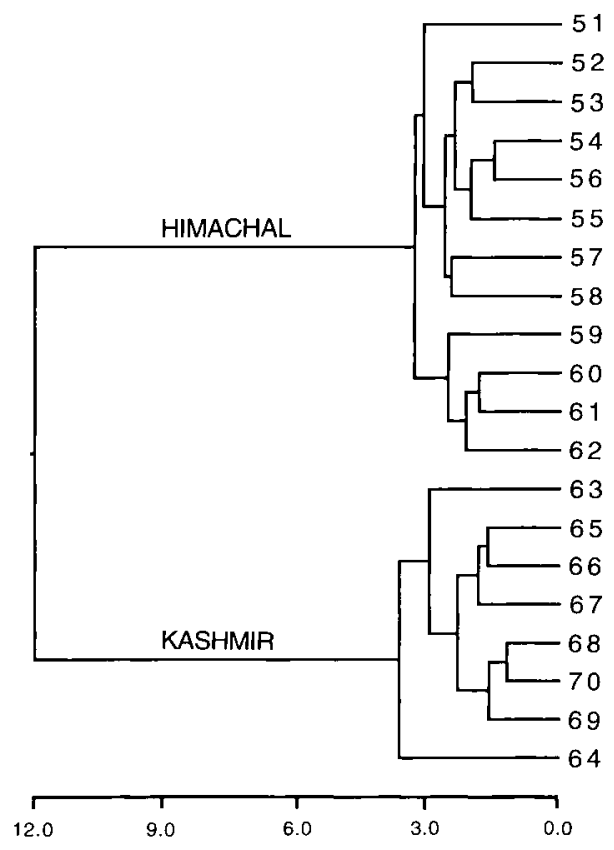

avec la pluviométrie négatives. C'est-à-dire qu'altitude élevée et pluviométrie faible sont corrélées avec une grande taille de l'abeille au sein de chaque région. Vingt-deux caractères sont uniformément corrélés avec l'altitude dans les 2 régions, mais seule la longueur de la partie apicale de la cellule radiale de l'aile antérieure est corrélée avec la pluviométrie dans les 2 régions. Le regroupement phénétique des échantillons au sein de chaque région correspond en général à la physiographie. L'analyse en composantes principales montre une contribution minime des composantes de la taille générale à la covariation globale de chacune des régions.

\section{Apis cerana / Himalaya / Himachal Pra- desh / Cachemire / morphométrie}

Fig 1. Phenogram from UPGMA cluster analysis of Euclidean distances between centroids representing samples from 20 localities. Site codes in table I. 'Himachal' and 'Kashmir' are biometric groups.

Abb 1. Phenogram der UPGMA Clusteranalyse von Euclidischen Abständen zwischen den Centroiden der Proben von 20 Orten. Die Ortcodes sind in Tabelle I. 'Himachal' und 'Kashmir' sind biometrische Gruppen. 


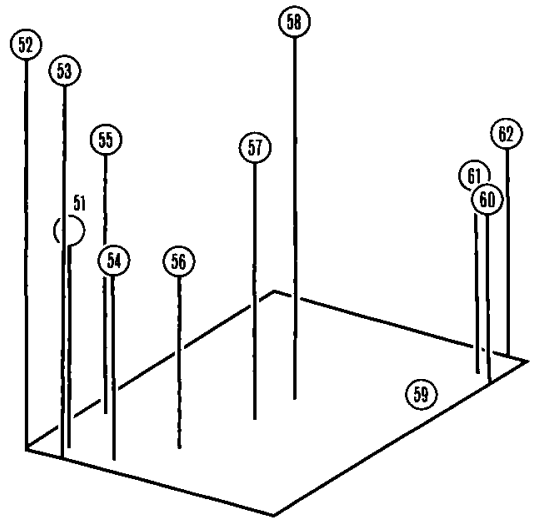

Fig 2. Three-dimensional views of the biometric relationships of samples of the Himachal region. Site codes for localities are in table I. Axes $X, Y$, and $Z$ are the first three canonical discriminant functions from analysis of the 20 samples that account for $90.6 \%, 2.6 \%$, and $1.2 \%$, respectively, of the total variation. Circles mark positions of centroids; diameter of circles is arbitrary.

$\boldsymbol{A} \boldsymbol{b} \boldsymbol{b}$ 2. Dreidimensionale Darstellung der biometrischen Ähnlichkeiten der Bienenproben aus der Himachalregion. Die Ortcodes sind in Tabelle 1. Die Achsen sind die ersten 3 canonischen Diskriminanzfunktionen aus der Analyse der 20 Proben. Sie repräsentieren $90,6 \%$ (X-Achse), $2,6 \%$ (Y-Achse) und 1,2\% (Z-Achse) der Gesamtvarianz. Die Kreise geben die Position der Zentroide an. Die Größe der Kreise ist willkürlich festgelegt.

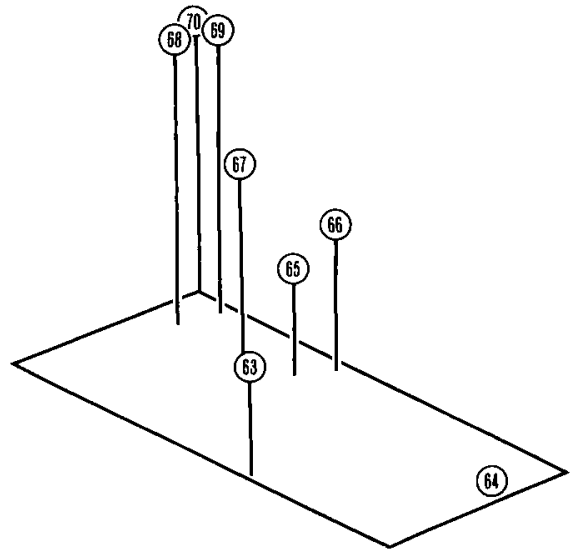

Fig 3. Three-dimensional views of the biometric relationships of samples of the Kashmir region. See for explanation legend to figure 2.

Abb 3. Dreidimensionale Darstellung der biometrischen Ähnlichkeiten der Bienenproben aus der Kashmirregion. Erläuterungen siehe Legende Abbildung 2. 
Table I. Code numbers of collection localities, geographic coordinates, altitude in meters (Alt), rainfall in millimeters (Rain), climatic classification of Takahashi and Arakawa (1981) (Clim; B = humid, C1 = dry sub-humid, $\mathrm{C}_{2}=$ moist sub-humid, $\mathrm{w}=$ rainfall deficient in winter, $\mathrm{s}=$ rainfall deficient in summer), and total number of bees collected from 4-5 wild colonies at each site (Bees).

Tabelle I. Codenummer des Sammelortes, geographische Koordinaten, Höhenlage in Metern (ALT), Niederschlagsmenge in $\mathrm{mm}$ (Rain), Klimatyp nach Takahashi und Arakawa (1981) (Clim; B = feucht, $C 1=$ trocken wenig feucht, $C_{2}=$ feucht bis wenig feucht, $w=$ Niederschlagsmangel im Winter, $s=$ Niederschlagsmangel im Sommer), und Anzahl aller an einem Ort von 4-5 wilden Völkern gesammelten Bienen (Bees).

Code Locality

Latitude

Biometric group of Himachal Region

$\begin{array}{ll}51 & \text { Bilaspur } \\ 52 & \text { Kangra } \\ 53 & \text { Mandi } \\ 54 & \text { Nahan (Sirmor) } \\ 55 & \text { Bhareri (Hamirpur) } \\ 56 & \text { Katrain (Kullu) } \\ 57 & \text { Solan } \\ 58 & \text { Dalhousi (Chamba) } \\ 59 & \text { Shimla (Simla) } \\ 60 & \text { Bagi (Shimla) } \\ 61 & \text { Poo (Kinnaur) } \\ 62 & \text { Roghi (Kinnaur) }\end{array}$

Biometric group of Kashmir region

$\begin{array}{ll}63 & \text { Rajouri } \\ 64 & \text { Kishtwar (Doda) } \\ 65 & \text { Srinagar } \\ 66 & \text { Kupwara } \\ 67 & \text { Tral (Pulwama) } \\ 68 & \text { Gurais (Baramulla) } \\ 69 & \text { Sonamarg (Srinagar) } \\ 70 & \text { Dras (Kargil) }\end{array}$

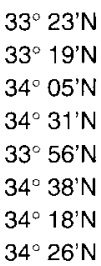

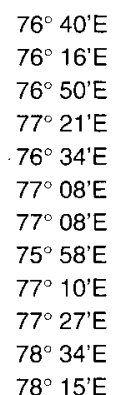

$78^{\circ} 15^{\prime} \mathrm{E}$

$\begin{array}{llrrll}31^{\circ} 15^{\prime} \mathrm{N} & 76^{\circ} 40^{\prime} \mathrm{E} & 587 & 1022 & \mathrm{Bw} / \mathrm{C}_{2} \mathrm{w} & 60 \\ 32^{\circ} 05^{\prime} \mathrm{N} & 76^{\circ} 16^{\prime} \mathrm{E} & 700 & 1623 & \mathrm{C}_{2} \mathrm{w} & 60 \\ 31^{\circ} 43^{\prime} \mathrm{N} & 76^{\circ} 50^{\prime} \mathrm{E} & 761 & 1388 & \mathrm{Bw} / \mathrm{C}_{2} w & 60 \\ 30^{\circ} 33^{\prime} \mathrm{N} & 77^{\circ} 21^{\prime} \mathrm{E} & 905 & 2221 & \mathrm{C}_{2} \mathrm{w} / \mathrm{C}_{1} \mathrm{~s} & 60 \\ 31^{\circ} 23^{\prime} \mathrm{N} & 76^{\circ} 34^{\prime} \mathrm{E} & 1007 & 1812 & \mathrm{C}_{2} \mathrm{w} & 60 \\ 32^{\circ} 05^{\prime} \mathrm{N} & 77^{\circ} 08^{\prime} \mathrm{E} & 1463 & 789 & \mathrm{Bw} / \mathrm{C}_{2} \mathrm{w} & 60 \\ 30^{\circ} 50^{\prime} \mathrm{N} & 77^{\circ} 08^{\prime} \mathrm{E} & 1530 & 1187 & \mathrm{Bw} / \mathrm{C}_{2} \mathrm{w} & 60 \\ 32^{\circ} 32^{\prime} \mathrm{N} & 75^{\circ} 58^{\prime} \mathrm{E} & 2036 & 2140 & \mathrm{C}_{2} \mathrm{w} & 60 \\ 31^{\circ} 07^{\prime} \mathrm{N} & 77^{\circ} 10^{\prime} \mathrm{E} & 2206 & 1625 & \mathrm{Bw} & 60 \\ 31^{\circ} 15^{\prime} \mathrm{N} & 77^{\circ} 27^{\prime} \mathrm{E} & 2648 & 1518 & \mathrm{Bw} & 60 \\ 31^{\circ} 40^{\prime} \mathrm{N} & 78^{\circ} 34^{\prime} \mathrm{E} & 2837 & 368 & \mathrm{Bw} & 60 \\ 31^{\circ} 32^{\prime} \mathrm{N} & 78^{\circ} 15^{\prime} \mathrm{E} & 3017 & 749 & \mathrm{Bw} & 60\end{array}$

Bees

$\begin{array}{rrll}938 & 1354 & C_{1} w & 60 \\ 1664 & 397 & C_{1} w & 60 \\ 1768 & 658 & C_{1} w & 60 \\ 1811 & 690 & C_{1} w & 60 \\ 2007 & 406 & C_{1} w & 60 \\ 2364 & 555 & C_{1} w & 60 \\ 2740 & 1815 & C_{1} w & 60 \\ 2977 & 500 & C_{1} w & 44\end{array}$


Table II. Code numbers for characters and brief descriptions of the measurements. Tergites and sternites are counted posterior to petiole, ie, as metasomal segments. Abbreviations refer to labels in illustrations of Mattu and Verma (1984a,b).

Tabelle II. Codenummern für die Merkmale und eine kurze Beschreibung der gemessenen Größen. Tergite und Sternite werden von vorne nach hinten gezählt. Die Abkürzungen beziehen sich auf die Beschriftung der Abbildungen von Mattu and Verma $(1984 a, b)$.

\section{Forewing}

1 Length of radial cell (RcL)

2 Breadth of radial cell ( $R c B$ )

3 Length of basal portion of radial cell $\left(R c L_{1}\right)$

4 Length of apical portion of radial cell $\left(\mathrm{RcL}_{2}\right)$

5 Length of forewing ( $F w L)$

6 Breadth of forewing (FwB)

7 Length of 1st abscissa of vein $\mathrm{M}_{3+4}$ in 2nd median cell (a)

8 Length of 2 nd abscissa of vein $\mathrm{M}_{3+4}$ in 2nd median cell (b)

9 Angle 31 of forewing

10 Angle 32

11 Angle 33

12 Angle 34

13 Angle 35

14 Angle 36

15 Angle 37

16 Angle 38

17 Angle 39

18 Angle 40

19 Angle 41

\section{Hindwing}

20 Length of basal portion of radial vein $(\mathrm{R} L)$

21 Length of apical portion of radial vein $(M L)$

22 Length of discoidal vein (VL)

23 Length of indica vein (IL)

24 Breadth of hind wing ( $\mathrm{HwB}$ )

25 Length of hind wing ( $\mathrm{HwL}$ )

26 Number of hamuli $(\mathrm{NH})$

27 Extent of hamuli (EH)

28 Length of jugal lobe (JL)

29 Length of vannal lobe (VL)
Hind leg

$\begin{array}{ll}30 & \text { Length of femur } \\ 31 & \text { Length of tibia } \\ 32 & \text { Length of metatarsus } \\ 33 & \text { Breadth of metatarsus }\end{array}$

Tongue

34 Length of prementum

35 Breadth of prementum

36 Total tongue length

37 Length of labial paip

38 Length of postmentum

39 Length of glossa

Abdomen (metasoma)

40 Length of light band of 3rd tergite

41 Length of dark band of 3rd tergite

42 Total length of 3rd tergite

43 Length of light band of 4 th tergite

44 Length of dark band of 4th tergite

45 Total length of 4 th tergite

46 Breadth of wax mirror on 3rd sternite $(\mathrm{WmB})$

47 Distance between wax mirrors on 3rd sternite (WmD)

48 Length of wax mirror on 3rd sternite (WmL)

49 Total length of 3rd sternite (S3)

50 Length or depth of 6th sternite (L6)

51 Breadth of 6th sternite (B6)

Antenna

52 Length of scape

53 Length of pedicel

54 Length of flagellum

55 Total length of antenna 
Table III. Means and standard errors for the 55 characters for each of the two biometric groups; means and standard deviations for the total sample. $n$, sample size.

Tabelle III. Mittelwert und Standardfehler für die 55 Merkmale der beiden biometrischen Gruppen; Mittelwerte und Standardabweichung für die gesamte Probe. $n$, Anzahl von Bienen der Probe.

\begin{tabular}{|c|c|c|c|c|c|c|}
\hline $\begin{array}{l}\text { Group: } \\
n=\end{array}$ & $\begin{array}{l}\text { Himach } \\
720 \\
\text { Mean }\end{array}$ & gion & $\begin{array}{l}\text { Kashmi } \\
464 \\
\text { Mean }\end{array}$ & $S E$ & $\begin{array}{l}\text { Total } \\
1184 \\
\text { Mean }\end{array}$ & $S D$ \\
\hline Character & & & & & & \\
\hline 1 & 3.012 & 0.0024 & 3.181 & 0.0038 & 3.078 & 0.1089 \\
\hline 2 & 0.403 & 0.0009 & 0.423 & 0.0012 & 0.411 & 0.0270 \\
\hline 3 & 1.198 & 0.0022 & 1.247 & 0.0021 & 1.217 & 0.0586 \\
\hline 4 & 1.850 & 0.0027 & 1.946 & 0.0031 & 1.888 & 0.0842 \\
\hline 5 & 8.498 & 0.0053 & 8.748 & 0.0071 & 8.596 & 0.1907 \\
\hline 6 & 2.847 & 0.0028 & 3.064 & 0.0034 & 2.932 & 0.1292 \\
\hline 7 & 0.537 & 0.0013 & 0.566 & 0.0017 & 0.548 & 0.0389 \\
\hline 8 & 0.143 & 0.0008 & 0.157 & 0.0011 & 0.148 & 0.0242 \\
\hline 9 & 32.182 & 0.0594 & 32.821 & 0.1124 & 32.432 & 1.9844 \\
\hline 10 & 107.499 & 0.1942 & 107.670 & 0.1811 & 107.566 & 4.7396 \\
\hline 11 & 93.778 & 0.1803 & 95.667 & 0.1757 & 94.518 & 4.5482 \\
\hline 12 & 19.261 & 0.0561 & 17.848 & 0.0935 & 18.707 & 1.8551 \\
\hline 13 & 88.365 & 0.1043 & 90.539 & 0.1344 & 89.217 & 3.0275 \\
\hline 14 & 43.472 & 0.1484 & 43.229 & 0.1690 & 43.377 & 3.8521 \\
\hline 15 & 98.174 & 0.1883 & 96.931 & 0.2171 & 97.687 & 4.9434 \\
\hline 16 & 82.333 & 0.1395 & 81.065 & 0.1736 & 81.836 & 3.7913 \\
\hline 17 & 15.035 & 0.0621 & 13.011 & 0.0718 & 14.242 & 1.8974 \\
\hline 18 & 74.622 & 0.1811 & 75.519 & 0.2090 & 74.974 & 4.7404 \\
\hline 19 & 31.332 & 0.0997 & 32.603 & 0.2643 & 31.830 & 4.1736 \\
\hline 20 & 1.440 & 0.0027 & 1.480 & 0.0024 & 1.456 & 0.0687 \\
\hline 21 & 1.221 & 0.0028 & 1.221 & 0.0033 & 1.221 & 0.0737 \\
\hline 22 & 1.196 & 0.0028 & 1.265 & 0.0038 & 1.223 & 0.0840 \\
\hline 23 & 0.386 & 0.0027 & 0.427 & 0.0036 & 0.402 & 0.0775 \\
\hline 24 & 1.653 & 0.0022 & 1.752 & 0.0026 & 1.692 & 0.0755 \\
\hline 25 & 5.791 & 0.0056 & 6.420 & 0.0066 & 6.037 & 0.3405 \\
\hline 26 & 19.360 & 0.0729 & 19.438 & 0.1008 & +9.390 & 2.0426 \\
\hline 27 & 1.241 & 0.0023 & 1.296 & 0.0029 & 1.262 & 0.0682 \\
\hline 28 & 1.748 & 0.0035 & 1.823 & 0.0052 & 1.778 & 0.1088 \\
\hline 29 & 1.204 & 0.0025 & 1.236 & 0.0029 & 1.216 & 0.0680 \\
\hline 30 & 2.468 & 0.0019 & 2.581 & 0.0035 & 2.512 & 0.0828 \\
\hline 31 & 3.094 & 0.0028 & 3.208 & 0.0035 & 3.139 & 0.0938 \\
\hline 32 & 1.941 & 0.0026 & 2.006 & 0.0039 & 1.967 & 0.0814 \\
\hline 33 & 1.086 & 0.0012 & 1.105 & 0.0029 & 1.093 & 0.0476 \\
\hline 34 & 1.313 & 0.0049 & 1.321 & 0.0060 & 1.316 & 0.1311 \\
\hline 35 & 0.782 & 0.0018 & 0.786 & 0.0025 & 0.784 & 0.0508 \\
\hline 36 & 5.307 & 0.0197 & 5.348 & 0.0247 & 5.323 & 0.5307 \\
\hline 37 & 2.552 & 0.0050 & 2.751 & 0.0120 & 2.630 & 0.2157 \\
\hline 38 & 0.302 & 0.0011 & 0.273 & 0.0017 & 0.291 & 0.0360 \\
\hline 39 & 2.776 & 0.0127 & 2.848 & 0.0157 & 2.804 & 0.3422 \\
\hline 40 & 1.028 & 0.0056 & 0.954 & 0.0077 & 0.999 & 0.1609 \\
\hline 41 & 0.781 & 0.0054 & 0.967 & 0.0055 & 0.854 & 0.1633 \\
\hline 42 & 1.977 & 0.0035 & 2.096 & 0.0023 & 2.024 & 0.0984 \\
\hline 43 & 0.657 & 0.0029 & 0.663 & 0.0043 & 0.659 & 0.0835 \\
\hline 44 & 1.097 & 0.0032 & 1.204 & 0.0040 & 1.139 & 0.1007 \\
\hline 45 & 1.899 & 0.0021 & 2.060 & 0.0038 & 1.962 & 0.1045 \\
\hline 46 & 2.003 & 0.0031 & 2.274 & 0.0029 & 2.109 & 0.1525 \\
\hline 47 & 0.210 & 0.0020 & 0.317 & 0.0025 & 0.252 & 0.0744 \\
\hline 48 & 1.520 & 0.0018 & 1.243 & 0.0032 & 1.412 & 0.1470 \\
\hline 49 & 2.429 & 0.0027 & 2.599 & 0.0028 & 2.495 & 0.1076 \\
\hline 50 & 2.325 & 0.0029 & 2.399 & 0.0042 & 2.354 & 0.0915 \\
\hline 51 & 2.589 & 0.0036 & 2.906 & 0.0053 & 2.714 & 0.1860 \\
\hline 52 & 1.079 & 0.0036 & 1.244 & 0.0039 & 1.144 & 0.1224 \\
\hline 53 & 0.238 & 0.0030 & 0.237 & 0.0012 & 0.237 & 0.0642 \\
\hline 54 & 2.536 & 0.0030 & 2.720 & 0.0034 & 2.608 & 0.1183 \\
\hline 55 & 4.109 & 0.0036 & 4.310 & 0.0035 & 4.188 & 0.1327 \\
\hline
\end{tabular}


Table IV. Left three columns are character codes and unstandardized and standardized discriminant function coefficients for the two biometric groups using all 55 characters. The values of the function at the group means are -4.2198 for the Himachal region and 6.5480 for the Kashmir region. Right two columns are character codes and pooled within-group correlations between the canonical discriminant function and the characters, ranked by the absolute magnitude of the correlations.

Tabelle IV. Die drei linken Spalten sind die Merkmalcodenummern, der Koeffizient der Diskriminanzfunktion und der standardisierte Koeffizient der Diskriminanzfunktion für die beiden biometrischen Gruppen unter Berücksichtigung aller Merkmale. Der Mittelwert der Funktion ist-4.2198 für die Himachalregion und 6.5480 für die Kashmirregion. Die beiden rechten Spalten sind die Merkmalcodenummer und die Korrelation innerhalb der gepoolten Gruppe zwischen der kanonischen Diskriminanzfunktion und dem Merkmal, geordnet nach der Größe der Korrelationen.

Unstandardized Standardized Correlations

Characters

Characters

$\begin{array}{lcr}1 & 2.645270 & 0.18796 \\ 2 & -1.703572 & -0.04302 \\ 3 & 0.5321126 & 0.02851 \\ 4 & 0.3717739 & 0.02595 \\ 5 & 0.5344067 & 0.07833 \\ 6 & 3.595537 & 0.26652 \\ 7 & 0.3461549 & 0.01251 \\ 8 & 0.9489423 & 0.02204 \\ 9 & -0.03228633 & -0.06330 \\ 10 & -0.002033616 & -0.00964 \\ 11 & 0.005976942 & 0.02663 \\ 12 & -0.06439338 & -0.11092 \\ 13 & 0.009580180 & 0.02717 \\ 14 & -0.01872391 & -0.07212 \\ 15 & -0.01755775 & -0.08617 \\ 16 & -0.02991329 & -0.11193 \\ 17 & -0.07899209 & -0.12799 \\ 18 & 0.004935637 & 0.02331 \\ 19 & 0.004977721 & 0.02055 \\ 20 & -0.1489012 & -0.00979 \\ 21 & -1.361788 & -0.10033 \\ 22 & 0.3238104 & 0.02491 \\ 23 & 0.03476011 & 0.00260 \\ 24 & 1.302947 & 0.07577 \\ 25 & 2.440815 & 0.35866 \\ 26 & -0.03021339 & -0.06173 \\ 27 & 0.8349808 & 0.05237 \\ 28 & -0.1195768 & -0.01226 \\ 29 & -0.3859466 & -0.02554 \\ 30 & 0.6083928 & 0.03760 \\ 31 & 1.515922 & 0.11469 \\ 32 & 0.2642043 & 0.01982 \\ 33 & -0.7396157 & -0.03454 \\ 34 & -0.5542313 & -0.07264\end{array}$

$\begin{array}{rr}48 & -0.45660 \\ 25 & 0.39747 \\ 46 & 0.33634 \\ 51 & 0.28480 \\ 6 & 0.27139 \\ 49 & 0.23174 \\ 54 & 0.22096 \\ 1 & 0.22086 \\ 45 & 0.21946 \\ 55 & 0.20830 \\ 47 & 0.18628 \\ 30 & 0.16939 \\ 52 & 0.16542 \\ 5 & 0.15842 \\ 24 & 0.15745 \\ 42 & 0.13975 \\ 31 & 0.13944 \\ 4 & 0.12862 \\ 41 & 0.12764 \\ 17 & -0.11601 \\ 44 & 0.11495 \\ 37 & 0.09588 \\ 3 & 0.08394 \\ 22 & 0.08337 \\ 50 & 0.08189 \\ 38 & -0.08171 \\ 27 & 0.08137 \\ 32 & 0.08006 \\ 12 & -0.07620 \\ 7 & 0.07586 \\ 2 & 0.07243 \\ 13 & 0.07116 \\ 28 & 0.06812 \\ 8 & 0.05737\end{array}$


Table IV continued.

\begin{tabular}{rrrrr} 
& Unstandardized & Standardized & \multicolumn{2}{l}{ Correlations } \\
35 & & & & \\
36 & -0.8156806 & -0.04146 & 20 & 0.05712 \\
37 & -0.09663903 & -0.05128 & 23 & 0.05119 \\
38 & 0.5239620 & 0.10097 & 29 & 0.04543 \\
39 & -6.485288 & -0.21451 & 40 & -0.04359 \\
40 & 0.1263396 & 0.04302 & 11 & 0.03937 \\
41 & -0.4190776 & -0.06575 & 33 & 0.03891 \\
42 & 0.1547772 & 0.02099 & 16 & -0.03149 \\
43 & 0.4589282 & 0.03641 & 9 & 0.03028 \\
44 & 0.9228163 & 0.07706 & 19 & 0.02860 \\
45 & -0.1136027 & -0.00980 & 15 & -0.02351 \\
46 & 3.089292 & 0.21145 & 39 & 0.01963 \\
47 & 4.704503 & 0.35313 & 18 & 0.01764 \\
48 & 3.799122 & 0.20204 & 35 & 0.00723 \\
49 & -8.540549 & -0.48283 & 36 & 0.00705 \\
50 & 2.885044 & 0.19703 & 43 & 0.00691 \\
51 & -0.4497991 & -0.03782 & 14 & -0.00586 \\
52 & 1.111705 & 0.11482 & 34 & 0.00542 \\
53 & 2.177542 & 0.20113 & 26 & 0.00354 \\
54 & 0.5788697 & 0.03718 & 10 & 0.00336 \\
55 & 3.235728 & 0.24982 & 53 & -0.00157 \\
& 1.763641 & 0.15781 & 21 & 0.00032
\end{tabular}

Constant-70.46841

Table V. Left three columns are character codes, unstandardized and standardized canonical discriminant function coefficients for the groups based on the best two variables. The values of the function at the group means are 2.53074 for the Himachal region and -3.927 for the Kashmir region. Right column is pooled within group correlations between the canonical discriminant functions and the characters.

Tabelle V. Die drei linken Spalten sind die Merkmalcodenummer, der Koeffizient der Diskriminanzfunktion und der standardisierte Koeffizient der Diskriminanzfunktion für die beiden biometrischen Gruppen unter Berücksichtigung der beiden besten Merkmale. Der Mittelwert der Funktion ist 2,53074 für die Himachalregion und $-3,927$ für die Kashmirregion. Die rechte Spalte ist die Korrelation innerhalb der gepoolten Gruppe zwischen der kanonischen Diskriminanzfunktion und den Merkmalen.

Unstandardized

Character

$25-4.413023$

$48 \quad 13.24839$

7.942608

Constant
Standardized

$-0.64847$

0.74898

\section{Correlations}

$-0.66275$

0.76134 
Table VI. Univariate ANOVAs and correlations of each of 55 characters. At left are character codes from table II. Univariate F ratio significance probabilities (FSIG) indicated by " $-"=>0.001$, ie, not significant, and by ${ }^{\text {*') }}=<0.001$, ie significant, for (1) ANOVA of 20 groups; (2) ANOVA of the 2 groups Himachal versus Kashmir regions; (3) ANOVA of 12 groups of Himachal region and (4) ANOVA of eight groups of Kashmir region. Correlations of morphometric characters of individual bees with altitude (ALT) and rainfall (Rain) within each of the biometric groups (Himachal region, Kashmir region). Only correlations with two-tailed significance probability $<0.01$ are given. $n$, sample size.

Tabelle VI. Univariate ANOVAs und Korrelationen für 55 Merkmale. Links sind die Merkmalcodenummern aus Tabelle II. Univariate F-Prüfzahl, Signifikanz-Niveau (FSIG): "-" = > 0.001 für nicht signifikant und "*' $=<0.001$ für signifikant. (1) ANOVA mit 20 Gruppen. (2) ANOVA mit zwei Gruppen, Himachalregion gegen Kashmirregion; (3) ANOVA mit 12 Gruppen der Himachalregion und (4) ANOVA mit 8 Gruppen der Kashmirregion. Korrelationen von morphometrischen Merkmalen von Einzelbienen mit Höhenlage (ALT) und Niederschlagsmenge (RAIN) innerhalb der biometrischen Gruppen (Himachalregion, Kashmirregion). Es werden nur Korrelationen angegeben, die mindestens eine zweiseitige Wahrscheinlichkeit von < 0.01 aufweisen. $n=$ Anzahl der Bienen pro Probe.

$\begin{array}{lll}\text { ANOVA } & \begin{array}{l}\text { Himachal region } \\ (n=720)\end{array} & \begin{array}{l}\text { Kashmir region } \\ (n=464)\end{array} \\ \text { FSIG } & \text { Alt } \quad \text { Rain } & \text { Alt }\end{array}$

Rain

\section{Forewing}

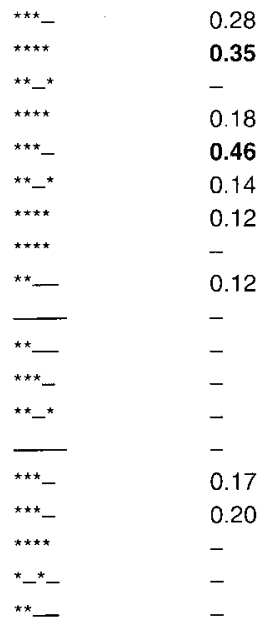

-0.13
-0.16
-
-0.15
-
-
-
-
-
-
-
-
-
-
0.11
-
-
0.10
-

0.19

0.18

$-$

0.13

0.13

0.23

0.30

0.27

0.13

$-$

0.12

0.18

$-$

$-$

0.19

-

$-$

\section{Hindwing}

$\begin{array}{llllll}20 & * * * & 0.16 & - & 0.17 & - \\ 21 & { }^{*}-{ }^{*}- & 0.19 & -0.14 & - & - \\ 22 & * \star- & - & - & - & - \\ 23 & { }^{* *} & 0.24 & -0.09 & - & - \\ 24 & { }^{* *}- & 0.33 & -0.16 & - & - \\ 25 & * *- & 0.14 & - & - & \end{array}$


Table VI continued

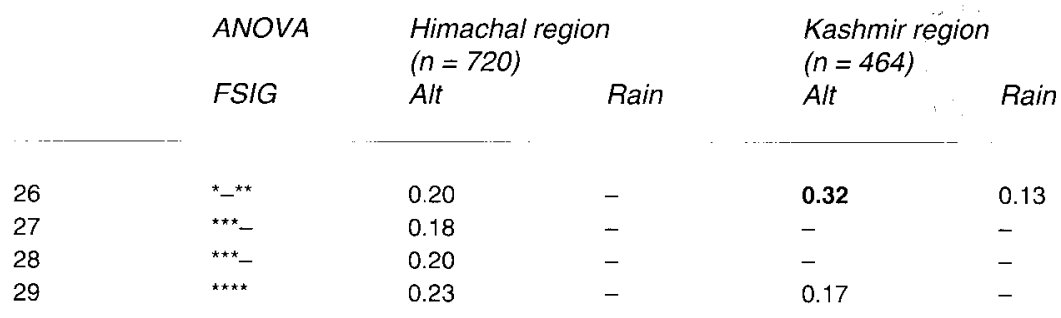

Hind leg

$\begin{array}{llllll}30 & { }^{* * *}- & 0.49 & -0.16 & 0.14 & - \\ 31 & { }^{* *}- & 0.17 & -0.10 & - & - \\ 32 & { }^{* * *}- & 0.25 & - & - & - \\ 33 & { }^{* *}- & 0.16 & - & 0.12 & -\end{array}$

Tongue

34
35
36
37
38
39

$\bar{T}$
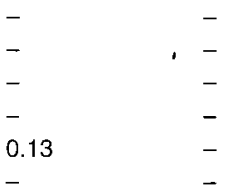

$\begin{array}{ll}- & - \\ - & - \\ - & - \\ - & - \\ 0.14 & - \\ 0.17 & -\end{array}$

Abdomen

\begin{tabular}{|c|c|c|c|c|c|}
\hline 40 & $* *-$ & -0.12 & - & -0.16 & - \\
\hline 41 & $* * \star \star *$ & 0.35 & -0.18 & 0.21 & - \\
\hline 42 & ${ }^{\star \star}-^{\star}$ & 0.17 & - & 0.25 & - \\
\hline 43 & ${ }^{*}$ - & -0.13 & - & -0.24 & - \\
\hline 44 & ${ }^{* \star *}-$ & 0.21 & -0.10 & 0.18 & - \\
\hline 45 & ${ }^{* *}-{ }^{*}$ & 0.12 & - & 0.26 & -0.13 \\
\hline 46 & ${ }^{* *} m^{*}$ & 0.11 & - & 0.12 & -0.12 \\
\hline 47 & ${ }^{* \star}-{ }^{*}$ & - & - & -0.16 & -0.27 \\
\hline 48 & ${ }^{* *}-$ & - & - & - & - \\
\hline 49 & $* \star *-$ & 0.15 & - & - & - \\
\hline 50 & ${ }^{\star \star \star}-$ & 0.39 & -0.19 & - & - \\
\hline 51 & ${ }^{\star * \star}-$ & 0.32 & -0.16 & 0.12 & - \\
\hline
\end{tabular}

Antenna

$\begin{array}{llllll}52 & * *- & - & - & - & - \\ 53 & - & - & - & - & - \\ 54 & { }^{* \star *} & 0.18 & - & - & - \\ 55 & * * *- & 0.19 & -0.10 & 0.11 & -\end{array}$


Table VII. Correlations of altitude (ALT), rainfall (RAIN) and 55 morphometric characters with principal components $1-3(\mathrm{PC} 1$, etc), and percent explained by each component $(\%)$. Correlations > absolute value of 0.3 in bold print. Analyzed separately for each region.

Tabelle VII. Korrelationen zwischen Höhenlage (ALT), Niederschlagsmenge (RAIN) und 55 morphometrischen Merkmalen und den Hauptfaktoren 1-3 (PC1, etc), sowie dem Prozentsatz der Gesamtvarianz (\%) die durch den jeweiligen Faktor erklärt wird. Korrelationen > als 0.3 (absoluter Wert) in Fettdruck. Die Analyse wurde für jede Region getrennt gerechnet.

Himachal region

\begin{tabular}{|c|c|c|c|c|c|c|}
\hline & PC1 & $P C 2$ & $P C 3$ & $P C 1$ & PC2 & PC3 \\
\hline$(\%)$ & 4.0 & 1.7 & 1.6 & 4.3 & 1.9 & 1.8 \\
\hline Alt & 0.872 & 0.054 & -0.090 & 0.560 & -0.503 & -0.115 \\
\hline Rain & -0.413 & 0.370 & -0.041 & 0.025 & -0.271 & 0.426 \\
\hline 1 & 0.312 & -0.092 & 0.067 & 0.409 & 0.092 & 0.020 \\
\hline 2 & 0.403 & 0.137 & -0.029 & 0.291 & -0.109 & -0.121 \\
\hline 3 & 0.121 & 0.149 & 0.138 & 0.213 & 0.255 & -0.247 \\
\hline 4 & 0.263 & 0.060 & 0.133 & 0.365 & 0.113 & -0.331 \\
\hline 5 & 0.486 & 0.281 & -0.048 & 0.320 & 0.018 & 0.110 \\
\hline 6 & 0.175 & 0.046 & -0.172 & 0.330 & -0.225 & 0.125 \\
\hline 7 & 0.179 & -0.044 & 0.381 & 0.331 & -0.264 & -0.047 \\
\hline 8 & 0.035 & 0.088 & 0.281 & 0.301 & -0.048 & -0.128 \\
\hline 9 & 0.161 & 0.115 & -0.246 & 0.330 & 0.182 & 0.098 \\
\hline 10 & 0.037 & -0.175 & 0.197 & 0.271 & 0.265 & -0.092 \\
\hline 11 & 0.081 & -0.189 & 0.107 & 0.288 & 0.254 & 0.196 \\
\hline 12 & 0.033 & 0.296 & -0.149 & 0.372 & 0.082 & 0.051 \\
\hline 13 & 0.062 & 0.111 & 0.086 & 0.263 & -0.044 & -0.210 \\
\hline 14 & 0.123 & -0.162 & 0.047 & 0.186 & 0.025 & -0.174 \\
\hline 15 & 0.271 & -0.304 & 0.183 & 0.203 & 0.215 & 0.012 \\
\hline 16 & 0.222 & 0.159 & -0.059 & 0.348 & 0.141 & 0.126 \\
\hline 17 & 0.072 & 0.051 & 0.222 & 0.270 & -0.188 & 0.090 \\
\hline 18 & 0.004 & -0.515 & 0.049 & 0.181 & 0.007 & -0.172 \\
\hline 19 & 0.089 & -0.021 & 0.013 & -0.049 & -0.185 & -0.082 \\
\hline 20 & 0.191 & -0.015 & -0.219 & 0.318 & -0.138 & 0.076 \\
\hline 21 & 0.264 & 0.049 & 0.137 & 0.268 & -0.031 & -0.040 \\
\hline 22 & 0.036 & 0.239 & 0.265 & 0.177 & -0.047 & -0.075 \\
\hline 23 & 0.272 & 0.072 & -0.110 & 0.276 & 0.052 & -0.123 \\
\hline 24 & 0.368 & 0.069 & -0.081 & 0.160 & 0.244 & -0.021 \\
\hline 25 & 0.170 & 0.265 & -0.401 & 0.225 & 0.000 & -0.171 \\
\hline 26 & 0.242 & 0.137 & 0.149 & 0.372 & -0.300 & 0.265 \\
\hline 27 & 0.239 & 0.209 & 0.088 & 0.177 & 0.104 & 0.113 \\
\hline 28 & 0.240 & 0.078 & -0.078 & 0.260 & 0.268 & 0.091 \\
\hline 29 & 0.309 & 0.163 & 0.289 & 0.341 & -0.128 & 0.231 \\
\hline 30 & 0.570 & -0.055 & -0.153 & 0.354 & -0.007 & 0.089 \\
\hline 31 & 0.313 & -0.104 & 0.240 & 0.329 & 0.281 & 0.023 \\
\hline 32 & 0.361 & -0.010 & 0.213 & 0.130 & 0.118 & 0.172 \\
\hline 33 & 0.254 & -0.119 & 0.246 & 0.332 & 0.104 & -0.204 \\
\hline 34 & 0.086 & 0.035 & 0.128 & 0.261 & -0.039 & 0.217 \\
\hline 35 & 0.083 & 0.080 & -0.046 & 0.142 & -0.140 & 0.105 \\
\hline 36 & 0.025 & -0.040 & -0.136 & 0.212 & 0.077 & -0.080 \\
\hline 37 & 0.037 & 0.137 & 0.033 & 0.101 & 0.013 & 0.160 \\
\hline 38 & 0.183 & 0.312 & 0.071 & 0.305 & 0.040 & 0.053 \\
\hline 39 & 0.030 & 0.029 & 0.283 & 0.191 & -0.184 & 0.381 \\
\hline 40 & -0.120 & 0.071 & 0.085 & 0.047 & 0.341 & 0.057 \\
\hline
\end{tabular}

Kashmir region 
Table VII. continued

\begin{tabular}{|c|c|c|c|c|c|c|}
\hline & \multicolumn{3}{|c|}{ Himachal region } & \multicolumn{3}{|c|}{ Kashmir region } \\
\hline & PC1 & PC2 & $P C 3$ & $P C 1$ & PC2 & $P C 3$ \\
\hline$(\%)$ & 4.0 & 1.7 & 1.6 & 4.3 & 1.9 & 1.8 \\
\hline 41 & 0.417 & -0.239 & -0.192 & 0.353 & -0.012 & -0.101 \\
\hline 42 & 0.215 & 0.008 & 0.019 & 0.422 & -0.106 & -0.085 \\
\hline 43 & -0.155 & 0.226 & 0.014 & -0.014 & 0.447 & 0.259 \\
\hline 44 & 0.290 & -0.136 & -0.100 & 0.318 & 0.081 & -0.288 \\
\hline 45 & 0.201 & -0.193 & -0.110 & 0.367 & -0.006 & -0.106 \\
\hline 46 & 0.155 & 0.236 & 0.245 & 0.243 & 0.047 & -0.144 \\
\hline 47 & 0.063 & -0.143 & 0.021 & $-0 . \uparrow 95$ & 0.371 & -0.033 \\
\hline 48 & 0.079 & -0.091 & 0.143 & 0.131 & 0.162 & 0.412 \\
\hline 49 & 0.207 & 0.379 & 0.023 & 0.244 & 0.237 & 0.132 \\
\hline 50 & 0.483 & -0.110 & -0.166 & 0.193 & 0.038 & 0.256 \\
\hline 51 & 0.388 & -0.142 & -0.208 & 0.151 & -0.109 & -0.304 \\
\hline 52 & 0.084 & -0.045 & 0.208 & 0.288 & 0.042 & 0.229 \\
\hline 53 & -0.005 & 0.145 & 0.125 & 0.125 & -0.177 & -0.087 \\
\hline 54 & 0.195 & -0.096 & 0.022 & 0.271 & 0.200 & -0.026 \\
\hline 55 & 0.235 & -0.099 & 0.027 & 0.202 & 0.044 & -0.058 \\
\hline
\end{tabular}

\section{REFERENCES}

Daly HV (1992) A statistical and empirical evaluation of some morphometric variables of honey bee classification. In: Ordination in the study of morphology, evolution and systematics of insects: applications and quantitative genetic rationals (JT Sorensen, $\mathrm{R}$ Foottit, eds) Elsevier, Amsterdam, 127-255

Mattu VK, Verma LR (1983) Comparative morphometric studies on the Indian honeybee of the North-west Himalayas. I. Tongue and Antenna. J Apic Res 22 (2), 79-85

Mattu VK, Verma LR (1984a) Comparative morphometric studies on the Indian honeybee of the North-west Himalayas. II. Wings. J Apic Res 23 (1), 3-10

Mattu VK, Verma LR (1984b) Comparative morphometric studies on the Indian honeybee of the North-west Himalayas. III. Hind leg, tergites and sternites. J Apic Res (23) 3, 117122

Norusis MJ (1985) SPSSX: Advanced statistics guide. McGraw-Hill Book Company, New York, $505 p$
Rohlf FJ (1987) Numerical taxonomy and multivariate analysis system for the IBM PC microcomputer (and compatibles). Applied Biostatistics, Inc, Setauket, New York, $36 \mathrm{p}$

Ruttner F (1988) Biogeography and taxonomy of honeybees. Springer-Verlag, Berlin, $284 p$

Singh MP, Verma LR, Daly HV (1990) Morphometric analysis of the Indian honeybee in the northeast Himalayan region. J Apic Res 29, 3-14

Sneath PHA, Sokal RR (1973) Numerical Taxonomy. WH Freeman and Company, San Francisco, $573 \mathrm{p}$

Takahashi K, Arakawa H (1981) Climates of Southern and Western Asia. In: World Survey of Climatology, vol 9 (HE Landsberg, ed) Elsevier Scientific Publishing Company, Amsterdam, $333 p$

Verma LR, Mattu VK (1982) Ecotypes studies on Apis cerana F. In: Biology of Social Insects (MD Breed, CD Michener, ME Evans, eds) West view Press, Boulder, Colorado, p 413

Verma LR, Mattu VK, Singh MP (1984) Races of Indian honeybee in Himalaya. Proc 15th Int Congr Entomol, Hamburg, p 508 